\title{
Avaliação de trialometanos formados na etapa de pré- oxidação com cloro e permanganato de potássio em água de abastecimento
}

\author{
Evaluation of trihalomethanes formed in pre-stage oxidation with \\ chlorine and potassium permanganate in supply water
}

\author{
Sabrina Firme Rosalém | Alexandre Demo Agrizzi \\ Márcia Cristina Martins Campos Cardoso | Edumar Ramos Cabral Coelho
}

Data de entrada: 05/10/2012 | Data de aprovação: 18/10/2012

Resumo

Este trabalho teve como objetivos identificar e quantificar trialometanos em água de abastecimento, a partir da água coletada do manancial Santa Maria da Vitória, com a intenção de simular as etapas de tratamento de água, inclusive a etapa de pré-oxidação com cloro ativo e permanganato de potássio. A determinação de trialometanos totais e o potencial de formação de TAMs, após o tratamento de água, foram realizados através da técnica de cromatografia gasosa com headspace-trap com detector de captura de elétrons. Com os resultados obtidos, foi possível comparar a formação dos trialometanos após o tratamento realizado com os dois oxidantes, revelando os benefícios do permanganato de potássio em relação ao cloro e, além disso, reforçando a idéia de que o permanganato de potássio representa uma boa opção de substituição.

Palavras-chave: cloro, permanganato de potássio, trialometanos, água de abastecimento.

\section{Abstract}

This work had as objectives to identify and quantify trihalomethanes in water supply, water collected from the wellspring of Santa Maria da Vitoria, with the intention of simulating the steps of water treatment, including the step of pre-oxidation with chlorine and permanganate potassium. The determination of trihalomethanes and the total formation potential TAMs after the water treatment was performed using gas chromatography with headspace-trap with an electron capture detector. With the results obtained, it was possible to compare the formation of trihalomethanes conducted after treatment with both oxidants, showing the benefits of potassium permanganate in respect to chlorine and furthermore reinforcing the idea that potassium permanganate is a good option substitution.

Key-words: chlorine, potassium permanganate, trihalomethanes, water supply.

Sabrina Firme Rosalém*

Química pela Universidade Federal do Espírito Santo (UFES). Mestre em Engenharia Ambiental pela Universidade Federal do Espírito Santo (UFES).

Alexandre Demo Agrizzi

Farmacêutico Bioquímico Industrial pela Universidade Federal de Ouro Preto (UFOP). Especialista em Química pela Universidade Federal de Lavras (UFLA). Mestre em Engenharia Ambiental pela Universidade Federal do Espírito Santo (UFES).

Márcia Cristina Martins Campos Cardoso

Química Industrial pela Universidade Vale do Rio Doce (UNIVALE). Mestre em Engenharia Ambiental pela Universidade Federal do Espírito Santo (UFES).

Edumar Ramos Cabral Coelho

Engenheira Civil pela Universidade Federal do Espírito Santo (UFES). Mestre e Doutora em Hidráulica e Saneamento pela Escola de Engenharia de São Carlos (EESC/USP). Professora adjunta do Departamento de Engenharia Ambiental da UFES.

* Endereço para correspondência: Centro Tecnológico - CT - UFES - Av. Fernando Ferrari, no 514, Campus Universitário Alaor Queiroz de Araújo, Goiabeiras, Vitória, Espírito Santo - Brasil - CEP 29075-910 - e-mail: binarosa@yahoo.com.br 


\section{Introdução}

Para atingir os padrões exigidos pela legislação brasileira (Portaria 518/2004 do Ministério da Saúde), a água captada de um manancial é submetida a vários processos de tratamento, como: pré-oxidação, coagulação, floculação, decantação, filtração e desinfecção. Duas etapas importantes são discutidas neste trabalho: a pré-oxidação e a desinfecção, devido à formação de subprodutos considerados de relevância para a saúde pública.

A oxidação química é utilizada para a remoção de matéria orgânica em estações de tratamento de água e nos tratamentos de águas residuárias industriais (TAN, 2005). Os oxidantes mais utilizados são o cloro gasoso ou seus derivados (hipoclorito de sódio ou hipoclorito de cálcio), devido seu baixo custo, fácil manuseio e alto potencial oxidante. $\mathrm{O}$ poder oxidante do cloro tem fornecido vários benefícios adicionais na etapa de pré-oxidação no tratamento de água, como a remoção de cor, ferro e manganês, assim como têm sido efetivo na redução de sabor e odor da água. No Brasil, muitas estações de tratamento de água utilizam o cloro como pré-oxidante. Essa prática tem sido questionada, devido à possibilidade de formação de subprodutos halogenados durante a cloração (PASCHOALATO, 2005).

Dentre os subprodutos originados da reação entre as substâncias húmicas e o cloro encontram-se os trialometanos (TAMs). Dentre os fatores que contribuem para a sua formação, podem-se citar: a concentração e composição da matéria orgânica natural, o tipo e a concentração de oxidante, a temperatura e o $\mathrm{pH}$ do meio.

Dos trialometanos possíveis de serem formados, quatro se formam em maior concentração, são eles: clorofórmio $\left(\mathrm{CHCl}_{3}\right)$, diclorobromometano $\left(\mathrm{CHBrCl}_{2}\right)$, dibromoclorometanto $\left(\mathrm{CHClBr}_{2}\right)$ e bromofórmio $\left(\mathrm{CHBr}_{3}\right)$. A soma das concentrações desses quatro compostos na água é chamada de trialometanos totais. Alguns estudos associaram a mortalidade por câncer com halometanos em água potável e relacionaram a presença desses compostos com vários tipos de câncer, incluindo o de bexiga e de cérebro (ANDREOLA et al., 2005).

No Brasil, a Portaria 518/2004 determina que o limite máximo permitido (LMP) de trialometanos totais para as águas de abastecimento público deve ser de $100 \mu \mathrm{g} \mathrm{L}^{-1}$. Esse valor coincide com o limite estabelecido pela União Européia, porém a tendência mundial é diminuir estes limites. Por isso, outros tipos de oxidantes passaram a ser estudados com o objetivo de substituir o cloro na etapa de pré-oxida- ção (CLEASBY et al., 1965).

Segundo a EPA, o permanganato de potássio pode ser utilizado para o controle de gosto e odor, remoção de cor, ferro, manganês e no controle do crescimento biológico nas estações de tratamento de água. Uma segunda aplicação do permanganato de potássio é o controle da formação de trialometanos (TAMs) e outros subprodutos (BRYANT, 1992).

\section{Objetivos}

Os objetivos deste trabalho estão focados na identificação e quantificação de trialometanos em água de abastecimento, levando-se em consideração a análise da realização da etapa de pré-oxidação utilizando o permanganato de potássio, combinado com o cloro, visando comparar as concentrações de subprodutos organoclorados formados a partir do uso destes oxidantes químicos.

\section{Metodologia}

Para o desenvolvimento deste trabalho foi utilizada água bruta coletada no manancial superficial Santa Maria da Vitória, que é utilizado para abastecimento público pela Companhia Espírito Santense de Saneamento (CESAN) e que abastece os municípios de Vitória e Serra, ambos no Espírito Santo. Os ensaios foram realizados em bancada, em teste de jarros, considerando a sequência: coagulação, floculação, decantação, filtração e desinfecção; precedida ou não da etapa de pré-oxidação.

O desenvolvimento do trabalho foi dividido em 06 (seis) etapas:

1. Caracterização da água bruta;

2. Elaboração do diagrama de coagulação, considerando a remoção de cor aparente;

3. Estudo da demanda de oxidantes, utilizando os compostos químicos permanganato de potássio e hipoclorito de cálcio para a determinação da dosagem do oxidante em relação ao tempo de contato para a pré-oxidação;

4. Determinação de trialometanos totais e do potencial de formação dos trialometanos, utilizando a técnica de Cromatografia Gasosa com headspace-trap (CG/HS-trap);

5. Realização das etapas do tratamento completo em teste de jarros: com e sem pré-oxidação, coagulação, floculação, sedimentação, filtração e desinfecção;

6. Avaliação sobre a influência do tratamento convencional na formação de trialometanos totais e no potencial de formação de TAMs, quando a pré-oxidação é realizada com o hipoclorito de cálcio e 
quando é realizada com permanganato de potássio.

A água bruta foi coletada e estocada em reservatório fechado, com ausência de luz, sob temperatura média de 220 C. A caracterização foi realizada através da medição dos parâmetros: $\mathrm{pH}$, turbidez, alcalinidade, absorbância em 254 nm, cor aparente e cor real, condutividade elétrica, análise de trialometanos totais e potencial de formação de trialometanos.

A determinação de cor aparente foi realizada através de leituras da absorbância da amostra de água bruta de acordo com o Standard Methods. As análises realizadas para elaboração do diagrama de coagulação foram feitas em bancada, utilizando reatores estáticos (jarteste). A etapa de pré-oxidação foi realizada com gradiente de velocidade de 100 $\mathrm{s}^{-1}$. Os oxidantes utilizados foram permanganato de potássio, $\mathrm{KMnO}_{4}$ (Synth, 99,0\% de pureza), e hipoclorito de cálcio, $\mathrm{Ca}(\mathrm{ClO})_{2}$ (CRQ, $65 \%$ de pureza).
Na Figura 01 estão representadas as etapas do estudo da pré-oxidação.

Para a detecção dos oxidantes foi utilizado o método de calibração externa, usando um espectrofotômetro da marca HACH DR 2000. Para a construção da curva analítica de calibração do permanganato de potássio foram realizadas leituras de absorbância de sete concentrações de $\mathrm{KMnO}_{4}$ que variaram de 0,5 a 3,5 $\mathrm{mg} \mathrm{L}^{-1}$ em meio ácido $(\mathrm{pH}<2,0)$ em comprimento de onda de $525 \mathrm{~nm}$, de acordo com Standard Methods $45000-\mathrm{KMnO}_{4} \mathrm{~B}$.

A curva de calibração externa do cloro ativo foi utilizada para o cálculo da concentração de cloro residual nos ensaios com hipoclorito de cálcio. $\mathrm{O}$ ensaio foi realizado com DPD, solução tampão de fosfato e oito concentrações diferentes de permanganato de potássio, que variaram de 0,0 a 3,5 mg $\mathrm{L}^{-1}$ a $515 \mathrm{~nm}$ de acordo com Standard Methods. A

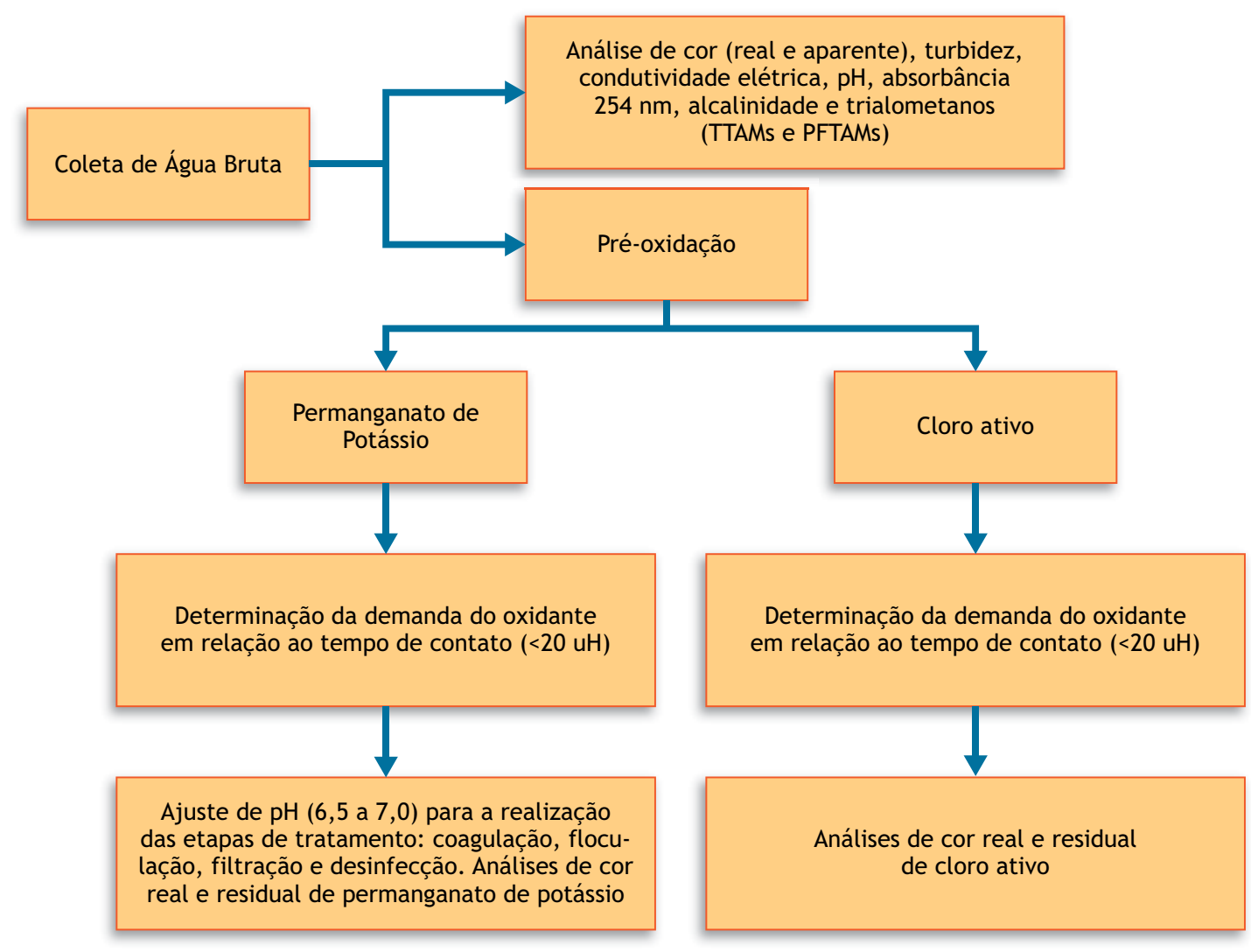

Figura 01: Etapas do estudo de demanda de pré-oxidantes

TTAMs: Trialometanos totais - PFTAMs: Potencial de formação de trialometano 
determinação da dosagem do oxidante e do tempo de contato para a oxidação foram definidos limitando-se a cor real para valores menores que 20 $\mathrm{uH}$, faixa que possibilita verificar a influência das etapas do tratamento de água na remoção da cor.

Os parâmetros para as etapas na simulação do tratamento da amostra de água bruta do manancial foram: pré-oxidação $\left(G=100 \mathrm{~s}^{-1} ; \mathrm{T}=10 \mathrm{~min}\right)$, seguida de coagulação utilizando sulfato de alumínio ( $\left.\mathrm{G}=500 \mathrm{~s}^{-1} ; \mathrm{T}=20 \mathrm{~s}\right)$; floculação $\left(\mathrm{G}=30 \mathrm{~s}^{-1} ; \mathrm{T}=\right.$ $20 \mathrm{~min})$; sedimentação (V = 1,0 cm/ $\left.\mathrm{min}^{-1}\right)$; filtração (filtro da marca Quality com porosidade de $28 \mu \mathrm{m}$ ) e a desinfecção utilizando cloro ativo com concentração de $6,0 \mathrm{mg} \mathrm{L}^{-1}\left(\mathrm{G}=100 \mathrm{~s}^{-1} ; \mathrm{T}=30 \mathrm{~min}\right)$.

Para avaliar a formação de trialometanos, foram propostas três rotas de tratamento. A primeira rota foi realizada sem a etapa da pré-oxidação; a segunda foi realizada com a etapa da pré-oxidação utilizando permanganato de potássio; e a terceira foi preparada com a etapa de pré-oxidação com hipoclorito de sódio. As análises de determinação de trialometanos foram realizadas em um Cromatógrafo a gás da Perkin Elmer modelo Clarus 500 acoplado a um sistema Headspace-trap e o detector utilizado foi DCE (Detector de Captura de Elétrons) instalado no laboratório da Fundação Nacional de Saúde - FUNASA/ES.

\section{Resultados e discussões}

Foram realizadas três coletas de água bruta durante o estudo de formação de trialometanos. As amostras de água da primeira coleta foram utilizadas para a elaboração do diagrama de coagulação; as amostras da segunda para o estudo da demanda dos oxidantes (permanganato de potássio e cloro ativo) e as da terceira, para a realização da simulação do tratamento convencional em Jarteste com a etapa de pré-oxidação e coleta para determinação de trialometanos totais e do potencial de formação de TAMs.

Após ter sido feita a caracterização físico-química da água bruta com uma das amostras, o diagrama de coagulação foi elaborado para auxiliar na escolha da dosagem de coagulante e alcalinizante na simulação do tratamento de água para abastecimento. A partir da análise do diagrama, a condição escolhida foi utilizando $10 \mathrm{mg} \mathrm{L}^{-1}$ de sulfato de alumínio em $\mathrm{pH}$ de 6,7. Esse ponto fornece uma cor aparente igual a $26 \mathrm{uH}$, necessitando realizar a etapa de pré-oxidação, para redução de cor.

O próximo passo foi efetuar os ensaios de demanda dos oxidantes, sendo inicialmente testando o permanganato de potássio. As dosagens aplicadas variaram de 0,25 a 2,0 mg. $\mathrm{L}^{-1} \mathrm{KMnO}_{4} \mathrm{em} \mathrm{pH}<2,0$ e as amostras foram retiradas nos tempos de contato: 10, 20, 30, 60 e 90 min. A dosagem escolhida de $\mathrm{KMnO}_{4}$ foi de $0,25 \mathrm{mg} \mathrm{L}^{-1}$ para a pré-oxidação em um tempo de contato de $10 \mathrm{~min}$, fornecendo um residual de $0,15 \mathrm{mg} \mathrm{L}^{-1} \mathrm{e}$ a cor real da água no final do processo foi de $5,8 \mathrm{uH}$.

Já no estudo da pré-oxidação da água utilizando o hipoclorito de cálcio, foram adicionadas concentrações iniciais de 0,25 a 2,5 mg. $\mathrm{L}^{-1}$ de cloro nos jarros. Após o estudo de oxidação com o cloro, a dosagem escolhida para a etapa de pré-oxidação foi de $1,0 \mathrm{mg} \mathrm{L}^{-1}$ em um tempo de contato de $10 \mathrm{~min}$, que gerou um residual de $0,08 \mathrm{mg} \mathrm{L}^{-1}$ de cloro ativo e cor de $17,5 \mathrm{uH}$.

Os valores de cor real da água bruta e da água tratada com pré-oxidação utilizando permanganato de potássio foram iguais. A medida da cor real da água bruta foi de 35,0 uH e após o tratamento convencional sem a etapa de pré-oxidação a cor obtida foi igual a $12,7 \mathrm{uH}$. A cor real obtida depois do tratamento convencional com a etapa de pré-oxidação utilizando permanganato de potássio e hipoclorito de cálcio foi 35,0 uH e 12,7 uH, respectivamente.

Os resultados indicam, portanto, que o tratamento convencional sem e com a pré-oxidação utilizando o cloro ativo forneceram o mesmo valor de cor real $(12,7 \mathrm{uH})$ a $465 \mathrm{~nm}$, o que sugere que o cloro ativo não foi eficiente na oxidação da matéria orgânica. No comprimento de onda de $254 \mathrm{~nm}$, para o tratamento utilizando o cloro ativo na etapa da pré-oxidação, mostrou-se mais eficiente do que o tratamento convencional sem a etapa e em relação à medida da absorbância, a amostra tratada com permanganato de potássio foi a que forneceu o menor valor de absorbância.

Na simulação do tratamento de água utilizando-se o permanganato de potássio na pré-oxidação, observou-se a dificuldade em controlar o $\mathrm{pH}$ durante o processo. A utilização do $\mathrm{KMnO}_{4}$ na pré-oxidação necessita de pH abaixo de 2,0 para que a oxidação da matéria orgânica seja eficiente (EPA, 1999). Para a redução do pH, foi utilizada solução de ácido sulfúrico 6,0 N. Para ajustar o pH para 7,0 (para a etapa de coagulação), após a oxidação, foi utilizada solução de hidróxido de sódio $0,1 \mathrm{~mol} \mathrm{~L}^{-1}$ ou ácido clorídrico $0,1 \mathrm{~mol} \mathrm{~L}^{-1}$.

Em relação aos trialometanos totais, os valores experimentais obtidos estão representados na Tabela 1.

A partir dos resultados obtidos, pode-se consta- 
tar que a maior formação de TAMs ocorreu quando a água foi tratada com pré-oxidação utilizando o cloro ativo, obtendo-se um valor igual a $57,5 \mu \mathrm{g}$ $\mathrm{L}^{-1}$. Quando o tratamento foi realizado sem a etapa de pré-oxidação, a concentração de TAMs formada foi igual a $48,5 \mu \mathrm{g} \mathrm{L}^{-1}$. A menor concentração de trialometanos detectados ocorreu na rota de tratamento utilizando-se permanganato de potássio, 26,5 $\mu \mathrm{g} \mathrm{L}^{-1}$. A formação de trialometanos totais foi 2,2 vezes maior no processo de tratamento uti- lizando cloro ativo na pré-oxidação em relação ao tratamento utilizando o permanganato de potássio. Para o rio Santa Maria da Vitória, em todas as rotas de tratamento realizadas, a formação das concentrações de TTAMs foram abaixo do limite estabelecido pela Portaria 518/2004, que é de $100 \mu \mathrm{g} \mathrm{L}{ }^{-1}$.

As amostras preparadas para a análise de potencial de formação de trialometanos foram analisadas no cromatógrafo a gás após sete dias de reação. Os resultados obtidos estão descritos na Tabela 2.

\begin{tabular}{c|c|c|c|c|c} 
Amostras & $\begin{array}{c}\text { Clorofórmio } \\
(\mu \mathrm{g}) / \mathrm{L}\end{array}$ & $\begin{array}{c}\text { Diclorobro- } \\
\text { mometano } \\
(\mu \mathrm{g}) / \mathrm{L}\end{array}$ & $\begin{array}{c}\text { Dibromoclo- } \\
\text { rometano } \\
(\mu \mathrm{g}) / \mathrm{L}\end{array}$ & $\begin{array}{c}\text { Bromofórmio } \\
(\mu \mathrm{g}) / \mathrm{L}\end{array}$ & TTHMs $(\mu \mathrm{g}) / \mathrm{L}$ \\
\hline $\mathrm{AB}$ & 0,1 & 0,3 & $\mathrm{ND}$ & 0,1 & 0,5 \\
\hline $\mathrm{SPO}$ & 9,0 & 29,0 & 9,0 & 2,0 & 48,5 \\
\hline $\mathrm{KMnO}_{4}$ & 8,0 & 12,0 & 5,0 & 2,0 & 26,5 \\
\hline $\mathrm{Cl}_{2}$ & 5,0 & 41,0 & 10,0 & 2,0 & 57,5 \\
\hline
\end{tabular}

AB: água bruta utilizada como branco;

SPO: rota de tratamento em que não foi realizada a pré-oxidação;

$\mathrm{KMnO}_{4}$ : rota de tratamento usando $\mathrm{KMnO}_{4}$ na pré-oxidação $\left(0,3 \mathrm{mg} \mathrm{L}{ }^{-1}\right)$;

$\mathrm{Cl}_{2}$ : rota de tratamento utilizando cloro ativo na pré-oxidação $\left(1,0 \mathrm{mg} \mathrm{L}^{-1}\right)$;

ND: não detectado.

Tabela 1: Resultados obtidos de trialometanos totais das amostras da água bruta e tratada do Rio Santa Maria da Vitória.

\begin{tabular}{c|c|c|c|c|c} 
Amostras & $\begin{array}{c}\text { Clorofórmio } \\
(\mu \mathrm{g}) / \mathrm{L}\end{array}$ & $\begin{array}{c}\text { Diclorobro- } \\
\text { mometano } \\
(\mu \mathrm{g}) / \mathrm{L}\end{array}$ & $\begin{array}{c}\text { Dibromoclo- } \\
\text { rometano } \\
(\mu \mathrm{g}) / \mathrm{L}\end{array}$ & $\begin{array}{c}\text { Bromofórmio } \\
(\mu \mathrm{g}) / \mathrm{L}\end{array}$ & $\begin{array}{c}\text { PFTHMs } \\
(\mu \mathrm{g}) / \mathrm{L}\end{array}$ \\
\hline $\mathrm{AB}$ & 0,1 & 0,3 & $\mathrm{ND}$ & 0,1 & 0,5 \\
\hline $\mathrm{SPO}$ & 4,0 & 30,0 & 11,0 & 3,0 & 47,5 \\
\hline $\mathrm{KMnO}_{4}$ & 7,0 & 12,0 & $\mathrm{ND}$ & 2,0 & 20,5 \\
\hline $\mathrm{Cl}_{2}$ & 7,0 & 45,0 & 12,0 & 2,0 & 65,5 \\
\hline
\end{tabular}

$\mathrm{AB}$ : água bruta utilizada como branco;

SPO: rota de tratamento em que não foi realizado pré-oxidação;

$\mathrm{KMnO}_{4}$ : rota de tratamento utilizando permanganato de potássio na pré-oxidação;

$\mathrm{Cl}_{2}$ : rota de tratamento utilizando cloro ativo na pré-oxidação;

ND: Não detectado

Tabela 2: Resultados obtidos de potencial de formação de trialometanos das amostras de água bruta e tratada do rio Santa Maria da Vitória 
A maior formação de PFTAMs também ocorreu quando a água foi tratada com pré-oxidação utilizando o cloro ativo, obtendo-se um valor igual a 65,5 $\mu \mathrm{g} \mathrm{L}^{-1}$. Quando o tratamento foi realizado sem a etapa de pré-oxidação, a concentração de trialometanos formados após 7 dias de reação foi igual a 47,5 $\mu \mathrm{g} \mathrm{L}^{-1}$. A menor concentração de trialometanos detectados ocorreu na rota de tratamento utilizando-se permanganato de potássio na pré-oxidação, 20,5 $\mu \mathrm{g} \mathrm{L}^{-1}$.

O potencial de formação de trialometanos foi 3,1 vezes maior no processo de tratamento utilizando cloro ativo na pré-oxidação em relação ao tratamento utilizando o permanganato de potássio. A formação de trialometanos foi 1,4 vezes maior quando o $\mathrm{Cl}_{2}$ foi utilizado na pré-oxidação, em relação ao tratamento sem pré-oxidação. A quantidade de trialometanos formados quando o tratamento foi realizado sem pré-oxidação foi 2,3 vezes maior do que no tratamento realizado com pré-oxidação utilizando o $\mathrm{KMnO}_{4}$.

\section{Conclusões}

Considerando a análise da etapa de pré-oxidação utilizando o permanganato de potássio, combinado com o cloro ativo, verificou-se que o permanganato de potássio mostrou ser mais eficiente que o cloro na remoção de cor real do manancial Santa Maria da Vitória. Além disso, apresentou-se ainda como melhor oxidante na remoção de absorbância a 254 $\mathrm{nm}$, trialometanos totais e potencial de formação de trialometanos, apesar da dificuldade em se controlar o $\mathrm{pH}$ durante o processo de tratamento.

Nas rotas de tratamento em que não foi realizada a etapa de pré-oxidação, os valores de TTAMs e PFTAMs foram superiores aos valores detectados quando foi utilizado permanganato de potássio na etapa de pré-oxidação.

Devido à tendência mundial de estabelecimento de padrões de qualidade da água mais restritivos e, considerando o menor valor limite encontrado na literatura, que é o estabelecido pela Itália de $30 \mu \mathrm{g}$ $\mathrm{L}^{-1}$, a rota de tratamento utilizando a pré-oxidação com permanganato de potássio foi a única que forneceu resultados de trialometanos com valores inferiores a este, demonstrando que para esse manancial o permanganato de potássio é uma alternativa de pré-oxidante que pode ser utilizada na etapa de pré-oxidação, substituindo o cloro e sem que haja comprometimento da saúde humana.

Esses resultados mostraram que o permanganato de potássio pode substituir o cloro na etapa de pré- -oxidação e assim, reduzir as concentrações de matéria orgânica natural antes da etapa de desinfecção (cloração).

\section{Referências}

ANDREOLA, R.; BERGAMASCO, R.; GIMENES, M. L.; FILHO, B. P. D.; CONSTANTINO, A. F. Formação de trialometanos em uma estação de tratamento de água. Acta Sci. Technol, v. 27, n. 2, p. 133-141, July-Dec., 2005.

APHA. Standard Methods for the Examination of Water and Wasterwater. 20th. ed. New York: American Public Health Association, 2000.

BRASIL. Leis decretos etc. In: Portaria 518. [S.1.]: Ministério da Saúde. Brasília, Brasil.

BRYANT, E. A.; FULTON, G. P.; BUDD, G. C. Disinfection Alternatives for Safe Drinking Water. [S.1.]: Van Nostrand Reinhold, 1992.

CLEASBY, J;; BAUMANN, E.; BLACK, C. Effectiveness of Potassium Permanganate for Disinfection. J. AWWA, p. 466-474, 1964.

DI BERNARDO, L.; DI BERNARDO, A.; CENTURIONE, P. Ensaios de tratabilidade de Água dos Resíduos Gerados em Estações de Tratamento de Água. [S.1.]: RiMa, 2002.

EPA. Alternative disinfectants and oxidants - potassium permanganate. In: EPA Guidance Manual - Chapter 5. 1999.

MEYER, S. T. O uso de cloro na desinfecção de Águas, a formação de trihalometanos e os riscos potenciais à saúde pública. Caderno de Saúde Pública, Rio de Janeiro, p. 99-110, 1994.

PASCHOALATO, C. F. P. R. Efeito da Pré-Oxidação, Coagulação, Filtração e Pós-Cloração na Formação de Subprodutos Orgânicos Halogenados em Águas Contendo Substâncias Húmicas. Tese (Doutorado) — Universidade de São Paulo, 2005.

SCHULZ, K., Dreßler J., Sohnius E., Lachenmeier D. W. Determination of volatile constituents in spirits using headspace trap technology. Journal of chromatography, p. 204-209, 2007.

TAN, Y. The removal of natural dissolved organic matter (dom) has been identified as one strategy to reduce the risk of disinfection byproducts (dbps) formed during the chlorination of natural water. Desalination 189-200, v. 176, p. 189-200, 2005. 